clinical states. It was demonstrated in polygraphic studies that the large plateau waves coincided with a reduction in local cortical blood flow and available oxygen. Clinical improvement after the administration of steroids was reflected in the record by the virtual disappearance of high pressure waves 12 hours after commencement of treatment. Similar records were obtained from patients with communicating hydrocephalus and 'benign intracranial hypertension'.

Eleven cases of so-called normal pressure hydrocephalus were monitored. The majority had flat pressure recordings but a few showed levels above normal at certain periods. Operation was performed in eight patients, half of whom subsequently showed improvement in intellectual performance. Two of these cases had suffered a previous subarachnoid haemorrhage.

The third group of cases were those with severe closed head injuries. Recordings were found to be particularly valuable where there was no evidence of an immediately remediable lesion since it was this group in which an insidious deterioration commonly occurred. On several occasions craniotomy had been undertaken because the recordings showed a rising ICP in the absence of clinical change. Examples were given in each group and the technical problems in relation to a fully reliable pressure transducer were described.

RELATIONSHIP BETWEEN INTRACRANIAL PRESSURE AND WATER CONTENT OF THE BRAIN IN EXPERIMENTAL BRAIN OEDEMA

J. W. F. BEKS and H. P. M. KERCKHOFFS (Groningen) had produced experimental cerebral oedema in cats by the application of cold to a circumscribed area of cortex through the intact dura. Four groups of animals were described.

Group $A$ consisted of controls. No lesion was inflicted and hyperosmotic solutions were not administered.

Group $B$ were killed when, after infliction of the lesion, intraventricular pressure had increased by $500 \mathrm{~mm} \mathrm{H}_{2} \mathrm{O}$ above its initial value. Hyperosmotic solutions were not administered.

Group $C$. The same procedure was followed, but when intracranial pressure reached $500 \mathrm{~mm} \mathrm{H}_{2} \mathrm{O}$ above its initial value a hyperosmotic urea solution was administered. The animals were killed at the lowest pressure subsequently achieved.

Group $D$ was the same as the preceding group, except for the substitution of a hyperosmotic mannitol solution for urea.
The results of this investigation showed:

1. There was no increase in water content of the injured portion of cerebral cortex compared with the uninjured cortex.

2. There was a significant increase in water content of the white matter underlying the injured cortex.

3. There was no decrease in cortical water content after administration of hyperosmotic solutions.

4. Dehydration of the abnormal white matter was more marked with urea than with mannitol.

5. A close correlation between cerebral water content and increase of intracranial pressure was not found.

\section{SOME ASPECTS OF SUBARACHNOID HAEMORRHAGE IN PREGNANCY}

J. L. ROBINSON and C. J. HALL (Liverpool) reviewed 26 cases of spontaneous subarachnoid haemorrhage which had occurred during pregnancy. Of the 24 lesions demonstrated to be responsible for the haemorrhages, 13 were aneurysms and 11 were arteriovenous malformations. Eight were aneurysms of the internal carotid artery and of these five were operated upon during pregnancy (four by a direct approach and one by carotid ligation). The remaining eight aneurysms and all the malformations were managed conservatively. Two of the malformations were subsequently treated surgically after recurrent haemorrhage. In the whole group there had been six previous episodes of subarachnoid haemorrhage in five patients, all with malformations. Only one of these had occurred unrelated to pregnancy. Of the 13 subsequent haemorrhages, seven occurred in seven patients with malformations, and six in five patients with aneurysms.

The authors had analysed their cases in conjunction with similar series in the literature and came to the following conclusions. A demonstrable cause of the haemorrhage was more likely than in non-pregnant cases and the incidence of aneurysms and malformations was about equal. The malformations were seen in younger women (20 to 25 ) and most commonly bled between 15 to 20 weeks. The aneurysms occurred in an older age group (30 to 35) and caused haemorrhage usually between 30 to 40 weeks, but rarely in labour or in the early puerperium. Maternal prognosis was the same as in non-pregnant cases, except that the malformations seemed to have a greater tendency to recurrent haemorrhage. The foetal prognosis was related to that of the mother in the case of aneurysm, but was unexpectedly poor in the malformation cases both in the current and in subsequent pregnancies. The possible causes of these differences were discussed and details of neurosurgical and obstetric management were given. 